The Astrophysical Journal Supplement Series, 90:631-638, 1994 February

(c) 1994. The American Astronomical Sociely. All rights reserved. Printed in U.S.A.

\title{
PULSED ACCELERATION IN SOLAR FLARES
}

\author{
MARKUS J. ASCHWANDEN \\ University of Maryland, Astronomy Department, College Park, MD 20742 \\ ARNOLD O. BENZ \\ Institute of Astronomy, Federal Institute of Technology (ETH), CH-8092 Zurich, Switzerland \\ BRIAN R. DENNIS \\ Laboratory for Astronomy and Solar Physics, NASA/Goddard Space Flight Center, Code 682, Greenbelt, MD 20771 \\ AND \\ MUKUl R. Kundu \\ University of Maryland, Astronomy Department, College Park, MD 20742 \\ Received 1993 January 25; accepted 1993 April 16
}

\begin{abstract}
We study the nonlinear dynamics of particle acceleration in solar flares by analyzing the time series of various quasi-periodic radio signatures during flares. In particular we present the radio and hard X-ray data of three flares which support the following tentative conclusions:

1. Particle acceleration and injection into magnetic structures occurs intrinsically in a pulsed mode (with a typical period of 1-2 s), produced by a single, spatially coherent, nonlinear system, rather than by a stochastic system with many spatially independent components ("statistical flare" produced by a fragmented primary energy release).

2. The nonlinear (quasi-periodic) mode of pulsed particle acceleration and injection into a coronal loop can be stabilized by phase locking with an MHD wave (oscillation) mode, if both periods are close to each other.

3. Pulsed injection of electron beams into a coronal loop may trigger nonlinear relaxational oscillations of wave-particle interactions. This is particularly likely when the limit cycles of both systems are similar.
\end{abstract}

Subject headings: acceleration of particles-Sun: corona - Sun: flares - Sun: radio radiation Sun: X-rays, gamma rays

\section{INTRODUCTION}

The temporal characteristics of particle acceleration, a most neglected aspect in solar flare literature, contains crucial information on the nonlinear dynamics of the dissipative system operating in the flare energy release process. A time series analysis reveals whether the dynamical system is driven by a harmonic oscillator (eigenmodes and strict periods in Fourier analysis), by deterministic chaos (with a low attractor dimension), or by independent stochastic processes (corresponding to an infinite attractor dimension). The time series analysis can also reveal important clues on the spatial organization of the dissipative system: deterministic systems with periodicities persistent over the duration of a flare are likely to be associated with a single spatially coherent system (e.g., a coherent electric field), while stochastic behavior indicates the superposition of a multitude of many spatially independent systems (e.g., wave turbulence). For solar flares, we have still no established information about the elementary organization of spatial and temporal scales during the energy dissipation process. Despite spatial high-resolution observations there is still no agreement about the spatial compactness ("compact flare") or the degree of fragmentation ("statistical flare") during the basic energy release.

Let us consider the nonlinear system dynamics of magnetic reconnection in solar flares for example. During the annihila- tion of magnetic energy in flares, the current-driven plasma instabilities can evolve into an oscillatory mode, where the current instability is coupled with the current sheet contraction by a feedback mechanism (Smith 1977), or the tearing mode can be driven into marginal stability (Spicer 1981). Sakai \& Ohsawa (1987) studied explosive reconnection driven by the current loop coalescence and simulated explosive and quasi-periodic particle acceleration. They applied this model to explain the quasi-periodic (double-peak) structures in some HXR flares (Kane et al. 1983; Forrest \& Chupp 1983; Nakajima et al. 1983; Kiplinger et al. 1983). Periodicities in solar type III bursts have been found in the range of 1-6 s (Mangeney \& Pick 1989) and have been interpreted in terms of modulation of the electron acceleration process (e.g., Carbone, Einaudi, \& Veltri 1987).

The basic dynamics of a dissipative system is either catastrophic or cyclic. If the system is near marginal stability, it can reveal periodic (nearly linear) oscillations, while the cycles are more irregular, but still deterministic, in the strongly nonlinear regime. Eventually the system may exhibit frequency doubling ("Feigenbaum route") and evolve on the route to chaos (e.g., "Hopf bifurcation," "Ruelle-Takens-Newhouse route"). Such a transition from a regular periodic to a chaotic system as part of the Ruelle-Takens-Newhouse route was identified in the temporal structure of the radio emission of the solar flare of 1980, April 3 (Kurths \& Karlicky 1989). A systematic analysis 
of the strange attractor dimension of decimetric broad-band pulsations, which are believed to be coherent emission from magnetically trapped particles, modulated either externally by MHD oscillations of the magnetic field (Roberts et al. 1984) or internally by self-organization (Aschwanden \& Benz 1988), revealed an information dimension in the range of 2.5-3.5 (Kurths, Benz, \& Aschwanden 1991). In contrast, the strange attractor dimension of decimetric millisecond spike emission was found to be large ( $\geq 5)$, indicating a stochastic or spatially highly fragmented system (Isliker 1992).

In this study we focus on those radio signatures that are the most sensitive tracers of particle acceleration in solar flares: propagating electron beams manifest as repetitive type III, type $\mathrm{J}$, or type U bursts. We report on three flares which show peculiar features that may lead to new insights of nonlinear system dynamics operating in the solar corona. In $\S 2.1$ we present a strictly periodic metric radio pulsation event which reveals inverted type $U$ structures, possibly indicating a phase-locking between quasi-periodic particle injection and a resonant magnetic loop, oscillating in an MHD mode (Fig. 1). In $\$ 2.2$ we present a typical flare with quasi-periodic trains of type III bursts and analyze their autocorrelation function to decide whether the system is deterministic or stochastic (Fig. 2). In $\S 2.3$ we show a flare which exhibits both metric quasi-periodic type III bursts and decimetric quasi-periodic broad-band pulsations, and study their mutual interaction and "mode coupling" (Fig. 3).

\section{OBSERVATIONS}

The observations were made with the film analog spectrograph DAEDALUS, and the digital radio spectrometer IKARUS of ETH Zurich (Perrenoud 1982). The analog data of DAEDALUS, recorded independently from IKARUS, were for the first time digitized here by microscanning of the film, having an original time resolution of $0.25 \mathrm{~s}$. IKARUS was operated with a time resolution of $0.1 \mathrm{~s}$ and a frequency resolution of $3 \mathrm{MHz}$ in the frequency range of $100-1000 \mathrm{MHz}$ in 1980. The hard X-ray observations were obtained with the Hard X-Ray Burst Spectrometer (HXRBS) of SMM (Orwig, Frost, \& Dennis 1980), with a time resolution of $0.128 \mathrm{~s}$ in the energy range of $25-450 \mathrm{keV}$. In the following we describe in detail three examples, selected from a set of 20 analyzed events.

\subsection{Periodically Driven Acceleration}

The flare of 1980 March 29, 1442 UT was described in several earlier studies under various aspects: to show the correlation of decimetric broad-band radio pulsations with the hard X-ray flux (Aschwanden, Benz, \& Kane 1990, their Fig. 3); the metric periodic oscillation between 300 and $340 \mathrm{MHz}$ at 1447:30 was used as a textbook example of an MHD oscillation mode in Roberts et al. (1984, their Fig. 6); the strange attractor dimension of the decimetric pulsations was determined to $D=3.4$, while the metric pulsation at $340 \mathrm{MHz}$ showed a dimension of $D=2.5$ (Kurths et al. 1991, their Fig. 1). We revisit this remarkable event under a new aspect which was not recognized before: the fine structure of the metric oscillation at $320-400 \mathrm{MHz}$ shows a striking "arcade-like" pattern between 1447:41 and 1447:51 UT. Figure 1 shows both the DAEDALUS records (with an rms sensitivity of $\approx 3$
SFU) and the IK ARUS records (with an rms sensitivity of $\approx 1$ SFU). Both records show clearly that the periodic pulses at 350 $\mathrm{MHz}$ have a faint extension at the high-frequency side, with a frequency-time drift rate that is dominantly negative, as typical for electron beams drifting upward in the solar corona (normal-drifting type III bursts). The IKARUS data show for some pulses that the upward-drifting structures turn over between 320 and $350 \mathrm{MHz}$ and form inverted U-type bursts, most clearly visible in the time period between 1447:41 and 1447:51 UT (Fig. 1, bottom). The descending leg of the U burst structures is generally weaker than the ascending leg, is sometimes disconnected from the previous ascending leg, and partially cancels the subsequent ascending leg.

Imaging observations suggest that inverted type $U$ bursts are plasma emissions of electron beams propagating in magnetic loop structures (Suzuki 1978). A study of a sequence of seven type-U bursts with the VLA has shown that the repetitive injection of electron beams occurs from the same stationary source location (Aschwanden et al. 1992a). A recent analysis of fine structures of the decimetric continuum during a flare revealed a quasi-periodic sequence of $\approx 45$ type $U$ burst segments, indicating pulsed injection with a mean period of $1.6 \mathrm{~s}$ (Aschwanden et al. 1993). If we interpret the "arcade-like" structure (Fig. 1) as sequence of $U$ bursts, this case represents the most regular periodicity for $U$ bursts ever reported. The Fourier spectrum shows a main period of $1.15 \mathrm{~s}$, and a secondary peak at $1.35 \mathrm{~s}$. The autocorrelation function (Fig. 4, top) exhibits a coherence length of $\approx 7 \mathrm{~s}$, which corresponds to the beat frequency of the two competing oscillation periods, similar to the case observed by Li, Messerotti, \& Zlobec (1987). The strict periodicity found in this sequence of $U$ bursts is certainly not typical for repetitive injection of electron beams, because the vast majority of quasi-periodic type III bursts ( see $\S 3.2$ ) is far less periodic and is more irregular in amplitudes. Thus we suggest that the strict periodicity is driven by an MHD oscillation mode of the magnetic loop, as proposed by Rosenberg (1970) and Roberts et al. (1984), rather than by a strictly periodic particle injection. However, the striking match between the MHD oscillation period and the duration of the underlying type U bursts suggests a "double resonance" between the mean periods of the accelerator and the oscillating flux tube. Since the quasi-periodic injection is generally less periodic, the "double resonance" may be interpreted as a case of phase-locking between a quasi-periodic accelerator and a (MHD-) resonant flux tube. The phase-locking is subject to a slow modulation of the beat frequency. The important lesson we can learn from this observation is that an MHD oscillation can stabilize the nonlinear modes of a quasi-periodic particle accelerator by phase locking. This is also indirect evidence for the pulsating nature of the accelerator, opposed to a stochastic system where phase locking cannot occur.

The interpretation in terms of $U$ bursts implies that the pulsating emission consists of plasma emission from periodically injected electron beams, opposed to the original interpretation of Rosenberg (1970) in terms of (MHD oscillation-) modulated gyrosynchrotron emission. Alternative models in terms of loss-cone-driven plasma instabilities periodically quenched by injected electron beams have been proposed by Benz \& Kuijpers (1976). The start frequencies of the $U$ bursts inferred here suggest that the pulsating accelerator is located near the 

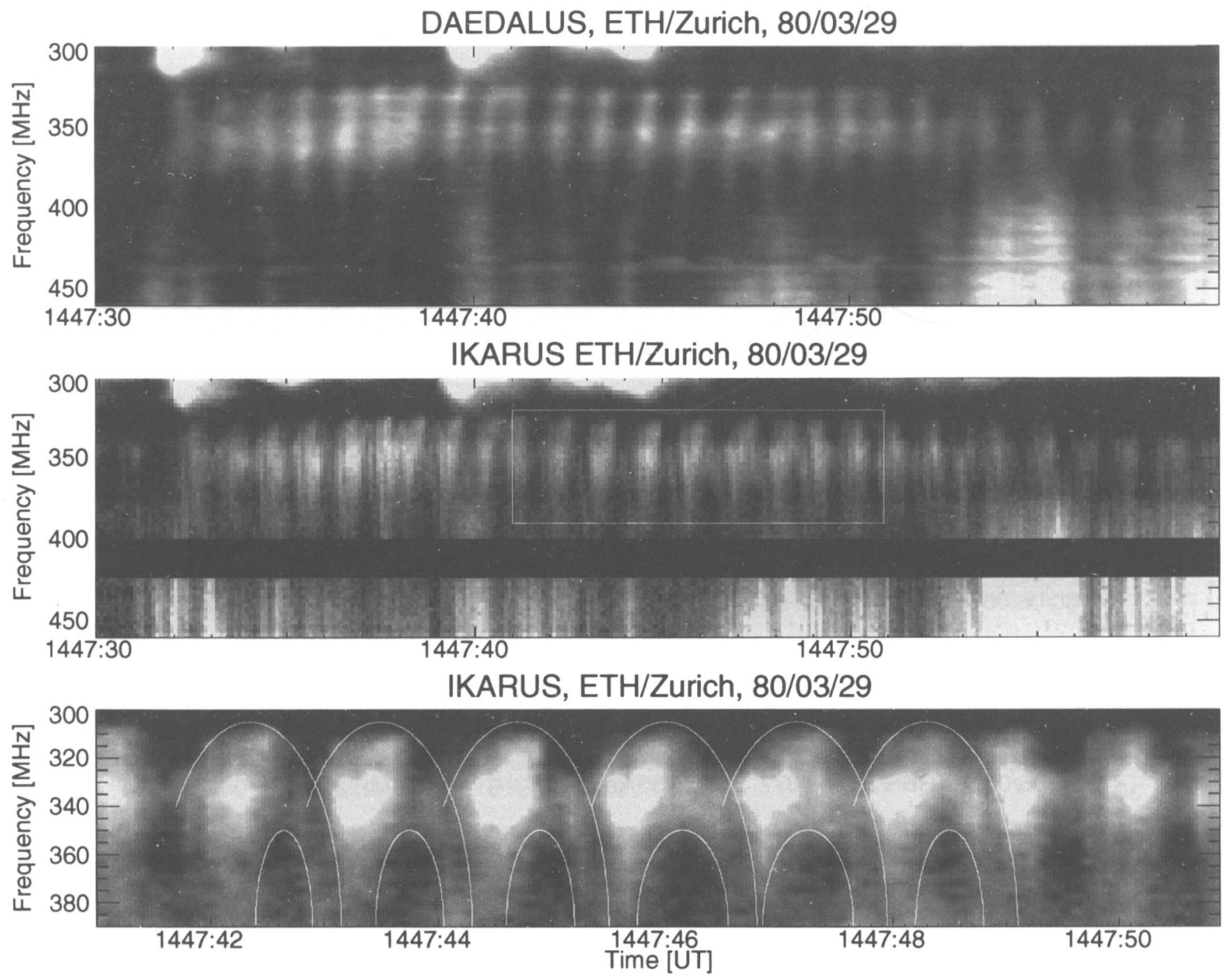

F1G. 1.-Periodic radio emission, recorded by the analog film spectrograph DAEDALUS (top) and digital spectrograph IK ARUS ( $m i d d l e$ and bottom) of ETH / Zurich on 1980 March 29, 1447:30-1448:00 UT, at 300-450 MHz. A 10 s segment (box in middle frame) is expanded in the bottom frame. White represents enhanced emission. A preflare background spectrum has been subtracted in the digitized DAEDALUS data to correct for the two disturbed frequencies at 390 and $440 \mathrm{MHz}$. The black bar at $400-420 \mathrm{MHz}$ in the IK ARUS data corresponds to a data gap. The pulsating structures show type III-like drifting structures, while some of the structures exhibit inverted U-type characteristics (contoured by white lines in the bottom frame). Note that the descending branch in the U-type structures is always weaker and interfers with the ascending branches.

footpoints of the oscillating loop, at an electron density of $\geq$ $10^{9} \mathrm{~cm}^{-3}$. The same basic scenario was inferred for the quasiperiodic sequence of 45 type-U burst segments (Aschwanden et al. 1993) and the first solar radio pulsation event detected by the VLA (Bastian, Aschwanden, \& Gary 1991; Aschwanden et al. 1992b). The detailed mechanism how an MHD oscillation modulates beam-driven plasma emission is not yet understood and poses an interesting problem in its own right, because MHD oscillations are expected to modulate chiefly the pitch angle or perpendicular velocity component of the particles, while the growth rate of Langmuir waves is mainly determined by the positive gradient of the parallel velocity component. The beam-driven radio emission could also be affected by whistler or upper hybrid waves, whose growth rate is very sensitive to the MHD-modulated pitch angle (Berney \& Benz 1978).

\subsection{Quasi-Periodic Injection from a Pulsed Accelerator}

In this section we describe the time behavior of particle acceleration in a typical flare by means of analyzing the time structure of type III radio bursts, the most unambiguous radio signature of electron beam acceleration. Figure 2 shows the radio and hard X-ray data from the flare 1980 June 6, 1550:401552:50 UT, a flare observed with good time and frequency (100-1000 MHz) coverage. The HXR time profile (Fig. 2, bottom) from HXRBS/SMM shows a weak flare with a peak count rate of 296 counts $\mathrm{s}^{-1}$ in the energy range of 25-450 $\mathrm{keV}$, exhibiting a preflare peak at $1550: 50$ and the main impulsive phase peaking at 1552:24 UT. While the HXR data show about five elementary structures with risetimes of $1-5 \mathrm{~s}$, more than 80 type III structures (with typical durations of $1 \mathrm{~s}$ ) can be counted in the contrast-enhanced dynamic spectrum shown in 

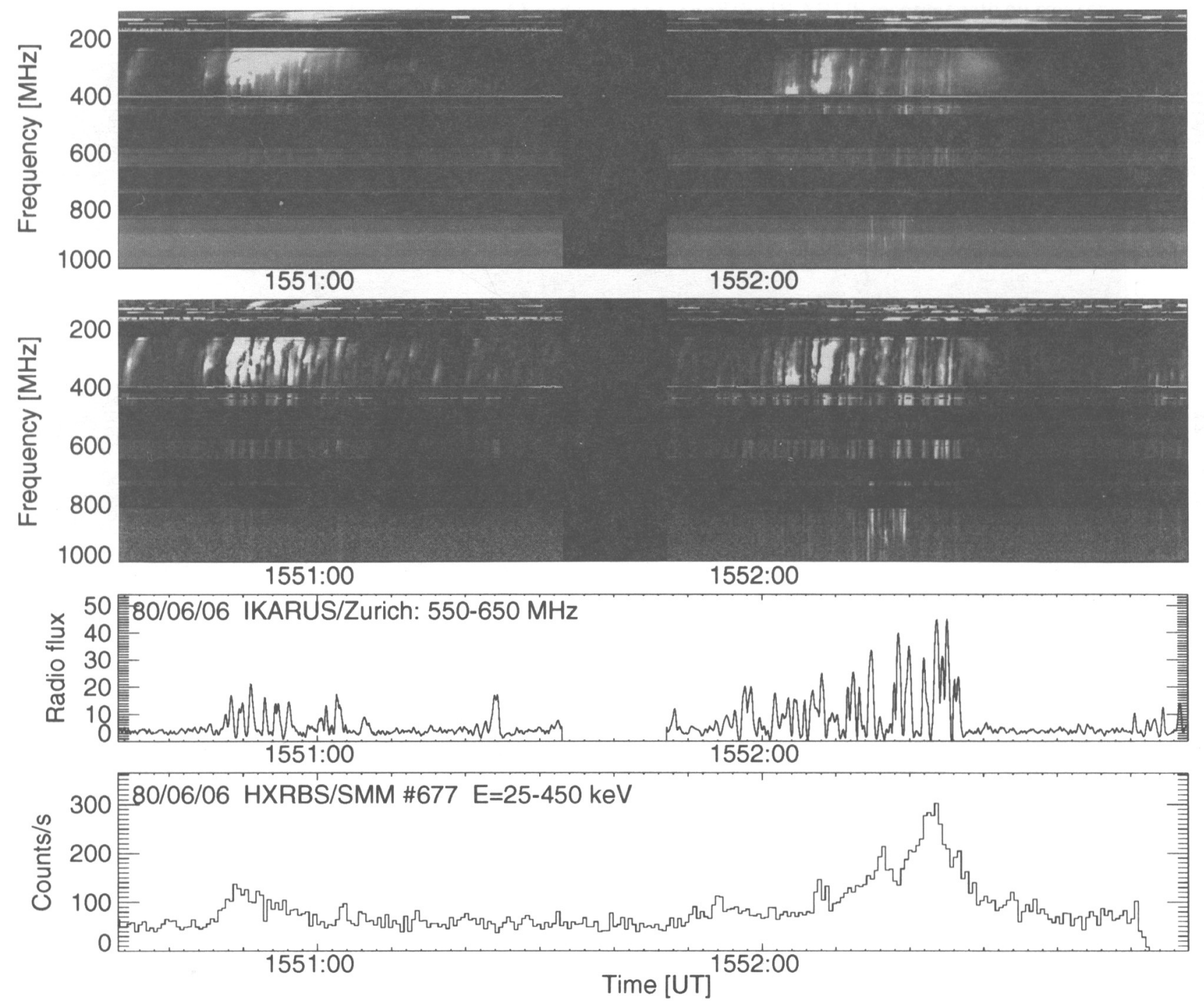

FIG. 2.-Quasi-periodic type III groups during the flare of 1980 June 6, 1551 UT. Top: Original IK ARUS radio data at 100-1000 MHz, Middle: envelope-subtracted IKARUS radio data at $100-1000 \mathrm{MHz}$. Second from bottom: envelope-subtracted radio data integrated over frequency range of 550-650 MHz. Bottom: hard X-ray data from HXRBS/SMM integrated with a time resolution of $0.512 \mathrm{~s}$ in the energy range of 25-450 keV.

Figure 2 (middle). The contrast-enhancement was performed by an envelope-subtraction technique described in Aschwanden et al. (1993). The typical time separation between subsequent type III bursts is $1-2 \mathrm{~s}$.

The time behavior of type III bursts is shown in Figure 2 ( second frame from bottom), revealing a train of quasi-periodic structures in the preflare phase (1550:45-1551:05), which become more erratic in the main flare phase (1551:501552:30). We show the autocorrelation function of this two time series in Figure 4; for the preflare phase (second from top), and during the main flare phase (third from top). The autocorrelation function in the preflare phase shows a sinusoidal variability with a mean period of $1.7 \mathrm{~s}$, indicating temporally coherent modulations, which would not occur in a stochastic system. In the main flare phase, the autocorrelation function reveals a more complex pattern, but is still governed by coherent modulations with periods of $1.3,1.7$, and $5.0 \mathrm{~s}$, as determined from FFT power spectra. It is striking that the basic period of $1.7 \mathrm{~s}$, is persistent during the entire flare.

An important question to understand the basic organization of the operating particle accelerator is whether the temporal dynamics is consistent with a single coherent system or with a spatially fragmented stochastic system. In a spatially fragmented stochastic system, individual energy dissipation processes are statistically independent, and thus incoherent, and the power spectrum of a corresponding time series is expected to show a $1 / \mathrm{f}$-noise spectrum, the autocorrelation function $A(d t)$ is expected to be close to zero for $d t \neq 0$, or the attractor dimension is expected to be infinite. Such a behavior was found for decimetric millisecond spikes (Isliker 1992). We show the autocorrelation function of such a time series with millisecond spikes in Figure 4 (bottom), where the amplitude 


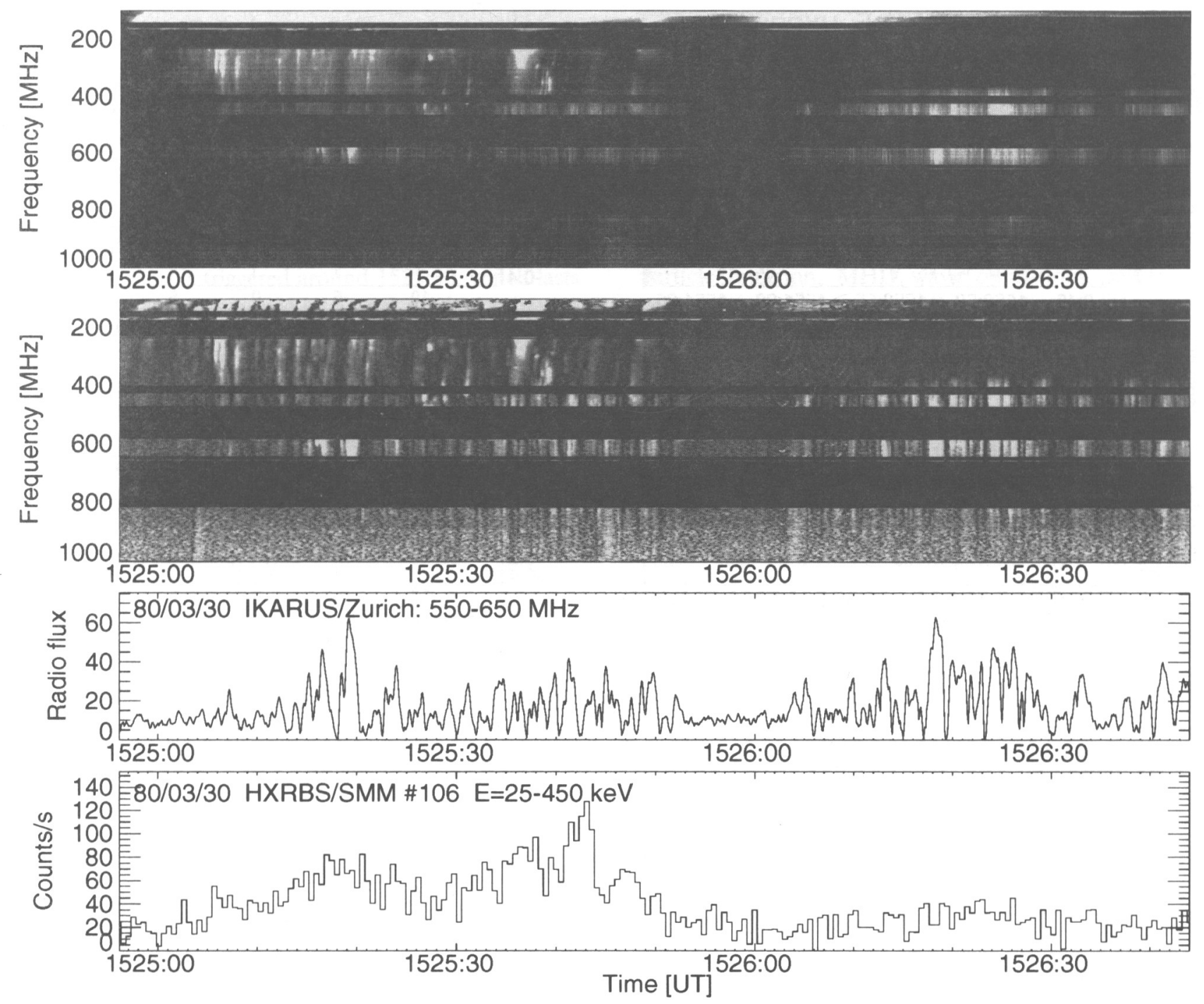

FIG. 3.-Quasi-periodic metric type III groups (at 100-300 MHz) and quasi-periodic decimetric broad-band pulsation ( $400-1000 \mathrm{MHz}$ ) during the flare of 1980 March 30, 1525 UT. Top: original IKARUS radio data at 100-1000 MHz. Middle: envelope-subtracted IKARUS radio data at $100-1000 \mathrm{MHz}$. Second from bottom: envelope-subtracted radio data integrated over frequency range of 550-650 MHz. Bottom: hard X-ray data from HXRBS/SMM integrated with a time resolution of $0.512 \mathrm{~s}$ in the energy range of $25-450 \mathrm{keV}$.

drops to zero for a time delay greater than the data resolution, as expected for a stochastic process.

Nonlinear dissipative systems are known to exhibit transitory and variable periods. If the attractor (orbit in phase space) of the nonlinear system is close to the limit cycle, the system exhibits a quasi-periodic oscillatory behavior. In the highly nonlinear regime, the periodicity can become quite complex, but the autocorrelation function is expected to show coherent modulations, in particular if the nonlinear system is near a limit cycle. Although there is a dynamical evolution toward more complexity during this flare, there is a persistent period of $\approx 1.7 \mathrm{~s}$ present during the entire flare, possibly representing the basic cycle of the nonlinear system. The rate of type III bursts does not significantly increase in the impulsive flare phase, but shows more complex dynamical behavior. Without analyzing the nonlinear dynamics in more detail (e.g., evolution of the strange attractor dimension during the flare, eventual indentification of a transition from a deterministic stage to a chaotic route), we conclude from the characteristics of the auto-correlation function and the persistent period of $\approx 1.7 \mathrm{~s}$, present during all flare phases, even after intermittent periods, that the time series of quasi-periodic type III bursts are consistent with the dynamical behavior of a single, spatially coherent, nonlinear system, rather than with a stochastic system with many spatially independent components. Since we found a similar dynamical behavior in 20 other flares we believe that particle acceleration in solar flares operates basically in a "pulsed mode," as opposed to continuous acceleration in a static DC field or stochastic acceleration in a spatially fragmented wave turbulence picture. The nonlinear period must 

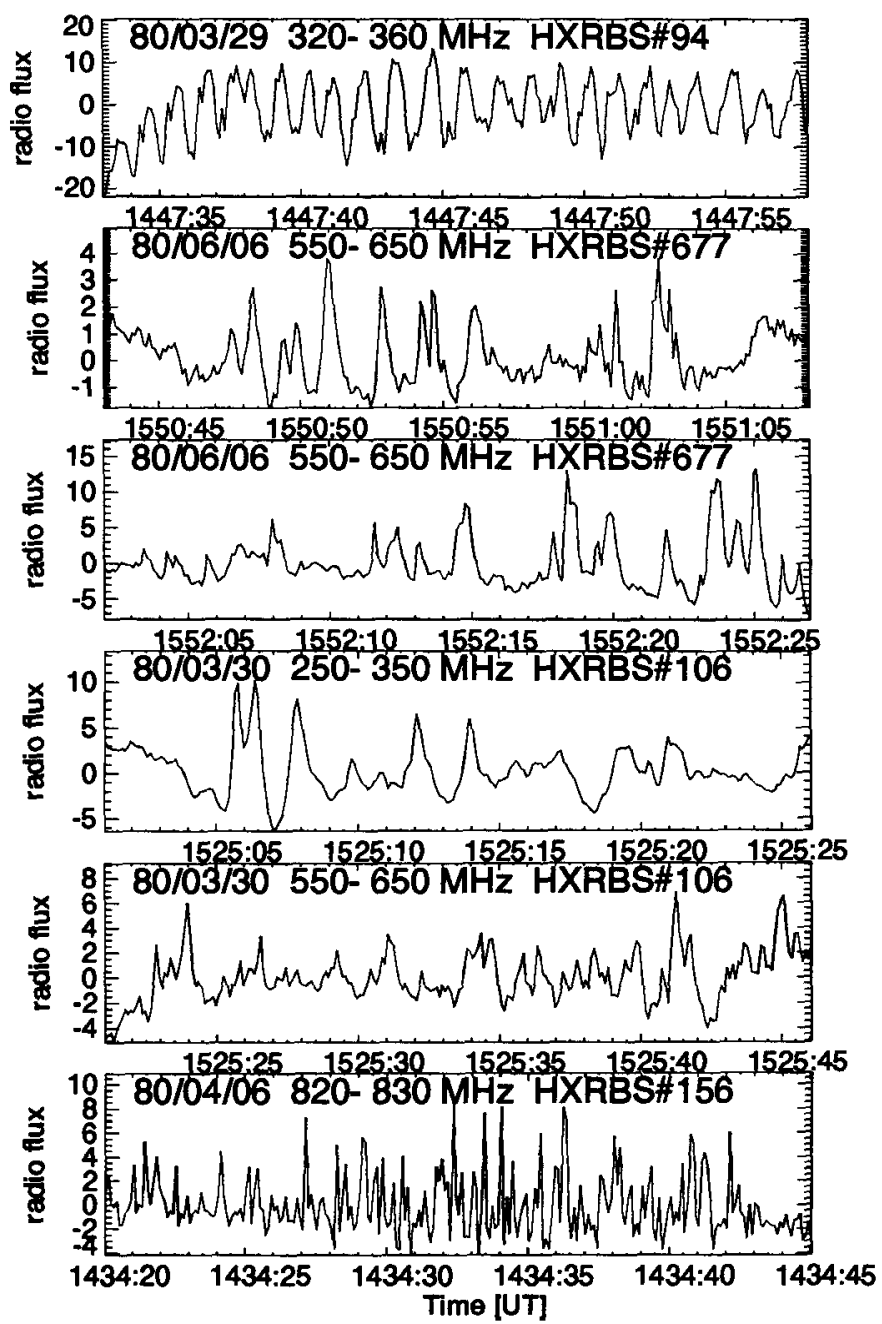
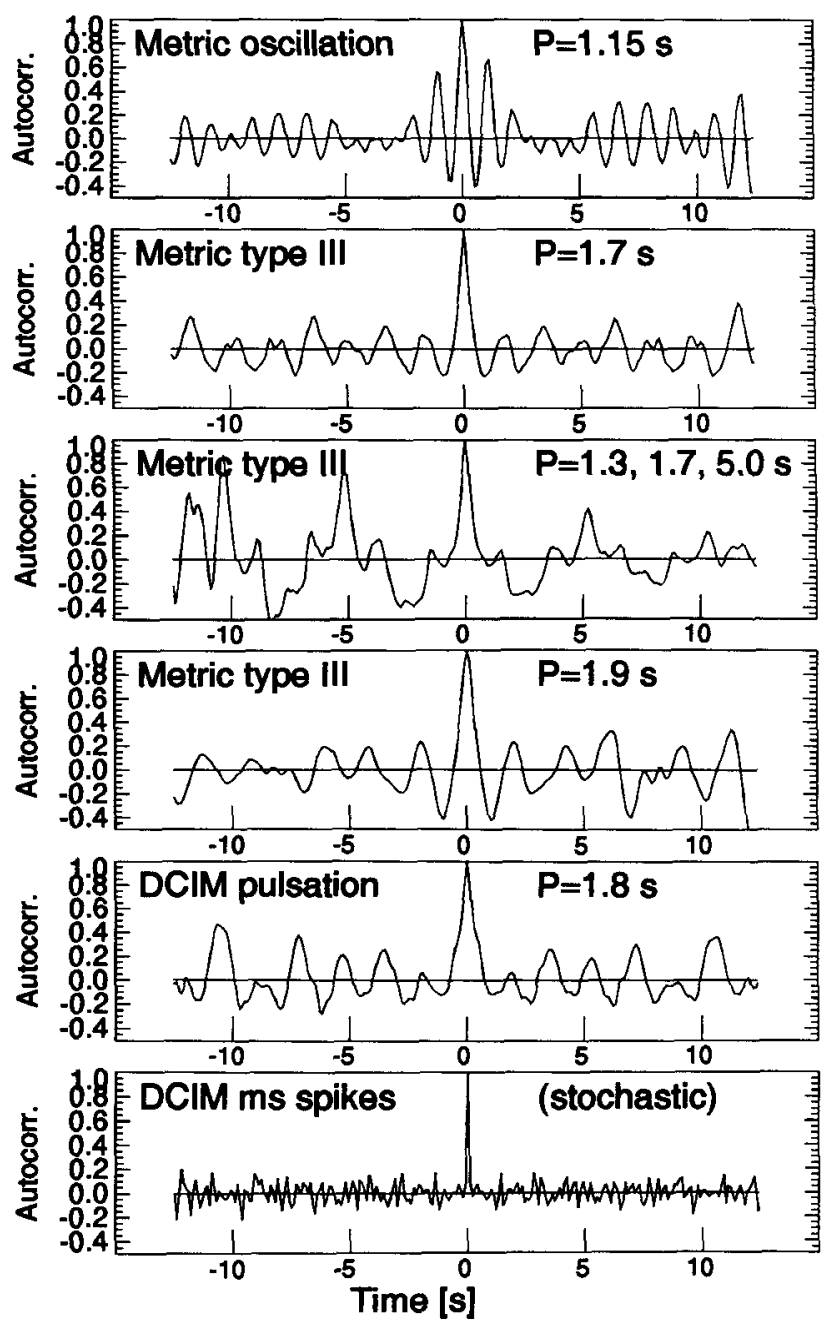

Fig. 4.-Six time series (left-hand side) extracted from the dynamic spectra shown in Figs. 1-3, and their autocorrelation function (right-hand side), after subtraction of a gliding background. Periodicities of coherent patterns in the autocorrelation function and also identified in FFT power spectra are indicated in seconds. Note the coherent patterns in the autocorrelation function in the upper 5 cases, compared with the stochastic case (bottom)

be a property of the accelerator itself rather than a property of a radiation-driven mechanism, because the periodicity of type III electron beams remains invariant over large $\left(\geq 10^{5} \mathrm{~km}\right)$ propagation distances.

\subsection{Response of a Coronal Trap to Pulsed Injection}

Pulsating solar radio emission is much more paramount in the decimetric range than at metric frequencies. The so-called decimetric (DCIM) quasi-periodic broad-band pulsations, reported by many observers (Young et al. 1961; Kundu 1965; Gotwols 1972; Elgarøy \& Sveen 1973; Bernold 1980; Wiehl et al. 1985; Aschwanden 1985; Aschwanden \& Benz 1986; Güdel \& Benz 1988) have generally been interpreted as coherent emission from trapped particles. The quasi-periodicity of the dynamical behavior has often been attributed to the nonlinearity of the dynamical system (see review of Aschwanden 1987 and references therein). The simplest form of a nonlinear system is a system of 2 coupled differential equations (e.g., LotkaVolterra equation), where a strictly oscillatory solution corre- sponds to the so-called "limit cycle," while irregular cycle periods indicate strong nonlinearity. If the dissipative system does not get unstable (which would result into a catastrophic behavior), it is likely to be driven into marginal stability, where external disturbances (initial conditions) can initiate nonlinear oscillations. However, the system behavior can not reliably be predicted without knowledge of the initial conditions, such as the dynamical aspects of the energy input, e.g., is the source function of nonthermal particles (which provide the free energy of the system), (1) continuous, (2) periodic or quasi-periodic, or (3) single-peaked (like a $\delta$-function in time)? Thus the time characteristics of particle acceleration is crucial to understand any secondary phenomena, such as beam-driven or loss-cone-driven plasma instabilities ( or other forms of coherent radio emission).

In the following we describe a flare where we can observe both the signatures of particle acceleration (type III groups) as well as the onset of a pulsating radio signature from the particle trap (DCIM broad-band pulsations). Figure 3 shows the flare of 1980 March 30, 1525:00-1526:40 UT. The hard X-ray data 
from HXRBS/SMM (Fig. 3, bottom) reveal that the impulsive phase takes place in the time interval of 1525:00-1525:55 UT, which is also coincident with the time interval of metric type III bursts. The auto-correlation function of the time series of metric type III bursts (Fig. 4, fourth row from top) shows some coherent modulations with an average period of $1.9 \mathrm{~s}$, indicating nonlinear cycles with transient periods. The phase of particle acceleration stops at 1525:55 UT, as indicated by the simultaneous end of metric type III bursts and impulsive HXR emission, but a decimetric broad-band pulsation with a mean period of $1.8 \mathrm{~s}$ is triggered around 1525:30, which lasts for more than $70 \mathrm{~s}$, accompanied by a weak gradual enhancement of HXR flux until 1526:40. The sharp low frequency cutoff (here at $380 \mathrm{MHz}$ ) is generally interpreted in terms of the apex height of the confining trap. Thus, the decimetric broad-band pulsation is likely to be produced by trapped particles, which get gradually lost from the trap and supply a weak precipitating electron flux, which is correlated with the gradual HXR component. The association of such a precipitation-induced HXR component with decimetric radio pulsations was studied for a larger number of flares in Aschwanden et al. (1990).

An interesting question is: How is the period of pulsed particle injection into the trap related to the nonlinear oscillation period of coherent radio emission from the trap? The autocorrelation functions show modulations with periods of $1.9 \mathrm{~s}$ for the metric type III bursts (Fig. 4; fourth frame from top), and periods of $1.8 \mathrm{~s}$ for the DCIM pulsations (Fig. 4; fifth frame from top). Thus, it is likely that the injection of particles with a mean period of $1.9 \mathrm{~s}$ triggers nonlinear oscillations of wave-particle interactions in the trap with a limit cycle of $1.8 \mathrm{~s}$. We analyzed six other flares with radio pulsations and found similar results: the mean period of type III bursts is often close to the mean period of decimetric broad-band pulsations, and decimetric broad-band pulsations usually follow an impulsive flare phase characterized by quasi-periodic type III bursts. Although type III bursts and decimetric broad-band pulsations share similar characteristics concerning their quasi-periodicity, they have quite different observational characteristics regarding frequency range, low-frequency cutoff, polarization (Aschwanden 1985), frequency-time drift rate (Aschwanden \& Benz 1986), and flare phase. Thus both phenomena are distinctly different types of radio emission, although their often simultaneous occurrence suggests an intimate coupling: The excitation of decimetric pulsations in the trap might crucially depend on the period of particle injection. Thus, pulsed beam injection (e.g., Karlicky 1990; Karlicky et al. 1990) should be seriously considered in trap models or trap-plus-precipitation models.

\section{CONCLUSIONS}

We analyzed the dynamical behavior of particle acceleration in three particular flares exhibiting three different types of quasi-periodic radio emission (type III groups, narrow-band metric oscillations, broad-band decimetric pulsations), which are believed to reflect three different physical processes (pulsed particle injection, MHD wave oscillations, self-organizing wave-particle interactions). We analyzed their periodicities and mutual interplays and find the following new insights, which are consistent with the findings obtained from the analysis of a larger set of flares described elsewhere:

1. Particle acceleration and injection into magnetic structures occurs intrinsically in a pulsed mode, produced by a single, spatially coherent, nonlinear system, rather than by a stochastic system with many spatially independent components. The spatial and temporal coherence of the dynamical system can be inferred from persistent periods and coherent modulations in the auto-correlation function, which do not occur in time series of stochastic systems (e.g., from decimetric millisecond spikes).

2. The quasi-periodic mode of electron beam injection into a magnetic loop can be stabilized by MHD oscillations (phaselocking). Although the detailed feedback mechanism is not understood, we found a flare where periodic radio signatures, commonly interpreted in terms of MHD oscillations, revealed distinguished features of type $U$ bursts.

3. Pulsed injection of electron beams into a coronal loop can trigger nonlinear relaxational oscillations of wave-particle interactions in a trap. It is conceivable that this trigger mechanism is most efficient when both systems have similar periods of their nonlinear cycles.

These conclusions should be considered as tentative because of the small number of presented examples, but studies with larger statistics are in progress (Aschwanden, Benz \& Montello 1994). The main purpose of this page-limited contribution is to give some stimulation to study the time characteristics of flare emission at all wavelengths under the important aspect of nonlinear system dynamics.

The work was partially funded by the grants NAS5-30442, NAGW-3080, and NAG 5-2001, and grant No. 20-34045.92 of the Swiss National Science Foundation.
Aschwanden, M. J. 1985, Sol. Phys., 104, 57 1987, Sol. Phys., 111, 113

Aschwanden, M. J., \& Benz, A. O. 1986, A\&A, 158, 102

- 1988, ApJ, 332, 466

Aschwanden, M. J., Benz, A. O., \& Kane, S. R. 1990, A\&A, 229, 206

Aschwanden, M. J., Benz, A. O., \& Montello, M. 1994, ApJ, submitted

Aschwanden, M. J., Bastian, T. S., Benz, A. O., \& Brosius, J. W. 1992a,

ApJ, 391, 380

Aschwanden, M. J., Bastian, T. S., \& Gary, D. E. 1992b, BAAS, 24/2, 802

Aschwanden, M. J., Benz, A. O., Dennis, A. O., \& Gaizauskas, V. 1993, ApJ, 416,857

\section{REFERENCES}

Bastian, T. S., Aschwanden, M. J., \& Gary, D. E. 1991, BAAS, 23/2, 1072

Benz, A. O., \& Kuijpers, J. 1976, Sol. Phys., 46, 275

Berney, M., \& Benz, A. O. 1978, A\&A, 65, 369

Bernold, T. E. X. 1980, A\&AS, 42, 43

Carbone, V., Einaudi, G., \& Veltri, P. 1987, Sol. Phys., 111, 31

Elgarøy, $\varnothing$, \& Sveen, O. P. 1973, Sol. Phys., 32, 231

Forrest, D. J., \& Chupp, E. L. 1983, Nature, 305, 291

Gotwols, B. L. 1972, Sol. Phys., 25, 232

Güdel, M., \& Benz, A. O. 1988, A\&AS, 75, 243

Isliker, H. 1992, Sol. Phys. 141, 325 
Kane, S. R., Kai, K., Kosugi, T., Enome, S., Landecker, P. B., \& McKenzie, D. L. 1983, ApJ, 271, 376

Karlicky, M. 1990, Sol. Phys., 130, 347

Karlicky, M., Alexander, D., Brown, J. C., \& MacKinnon, A. L. 1990, Sol. Phys., 129, 325

Kiplinger, A. L., Dennis, B. R., Frost, K. J., \& Orwig, L. E. 1983, ApJ, 273, 783

Kundu, M. R. 1965, Solar Radio Astronomy (New York: Interscience)

Kurths, J., Benz, A. O., \& Aschwanden, M. J. 1991, A\&A, 248, 270

Kurths, J., \& Karlicky, M. 1989, Sol. Phys., 1 19, 399

Li, H.-W., Messerotti, M., \& Zlobec, P. 1987, Sol. Phys., 111, 137

Mangeney, A., \& Pick, M. 1989, A\&A, 224, 242
Nakajima, H., Kosugi, T., Kai, K., \& Enome, S. 1983, Nature, 305, 292 Orwig, L. E., Frost, K. J., \& Dennis, B. R. 1980, Sol. Phys., 65, 25 Perrenoud, M. 1982, Sol, Phys., 81, 197

Roberts, B., Edwin, P. M., \& Benz, A. O. 1984, ApJ, 279, 857

Rosenberg, H. 1970, A\&A, 9, 159

Sakai, J.-I., \& Ohsawa, Y. 1987, Space Sci. Rev., 46, 113

Smith, D. F. 1977, J. Geophys. Res., 82/4, 704

Spicer, D. S. 1981 , Sol. Phys., 70, 149

Wiehl, H. J., Benz, A. O., \& Aschwanden, M. J. 1985, Sol. Phys., 95, 167 Young, C. W., Spencer, C. L., Moreton, G. E., Roberts, J. A., \& Kundu, M. R. 1961, ApJ, 133, 342 